\title{
Nodular vasculitis associated with lung adenocarcinoma*
}

\author{
Ana Sofia Borges ${ }^{1}$, Ana Brasileiro ${ }^{1}$, Margarida Apetato ${ }^{1}$
}

DOI: http:/ / dx.doi.org/10.1590/abd1806-4841.20187741

\begin{abstract}
Nodular vasculitis is an uncommon type of panniculitis. It was firstly associated to the infection by Mycobacterium tuberculosis and named erythema induratum of Bazin, however nowadays it has been associated with several other underlying disorders and it is better described as nodular vasculitis. Concerning its relationship with malignant conditions, there is only one reported case, in association with metastatic colon adenocarcinoma. We report a case of nodular vasculitis as the first manifestation of lung adenocarcinoma. To the best of our knowledge, this is the first reported association of both diseases.
\end{abstract}

Keywords: Lung neoplasms; Panniculitis; Paraneoplastic syndromes

\section{INTRODUTION}

Nodular vasculitis (NV) is an uncommon form of panniculitis characterized by erythematous nodules or plaques located preferentially on the calves, which may ulcerate and drain. It has been regarded as a delayed hypersensitivity response to an antigenic stimulus, being originally associated with tuberculosis. ${ }^{1}$ However, some cases in association with both non-tuberculous infections, such as hepatitis B and C virus, ${ }^{1-2}$ and non-infectious conditions, such as autoimmune diseases, ${ }^{2}$ inflammatory bowel disease ${ }^{3}$ and drugs $^{4}$ were reported. NV has rarely been associated with malignant diseases, with only one case reported in the literature related to metastatic colon adenocarcinoma. ${ }^{5}$ Histopathologic features include a septal/lobular panniculitis with a mixed inflammatory infiltrate containing lymphocytes, plasm cells, histiocytes forming granulomas, neutrophils, eosinophils and vasculitis. ${ }^{6}$

\section{CASE REPORT}

A 63-year-old woman was seen with tender, erythematous lesions on both legs that appeared 8 days before. Patient denied other symptoms such as fever, weight loss, asthenia or cough. Past medical history included arterial hypertension, type 2 diabetes mellitus, acute ischemic stroke without sequelae and venous insufficiency of lower limbs. She was not a smoker. Her brother was a heavy smoker and had had lung cancer.

Physical examination revealed a symmetrically distributed dermatosis, with several ill-defined non-ulcerated bilateral tender erythematous subcutaneous nodules with 2-4 cm located on the legs (Figure 1). On histopathologic examination, there was a predominantly lobular panniculitis with vasculitis and necrosis (Figure 2A).
The inflammatory infiltrate contained mononuclear cells and neutrophils (Figure 2B).

Complementary investigation was performed, with a negative tuberculin skin test; on chest X-ray a suspicious pulmonary nodule was observed, which presence was confirmed by computed tomography. A transbronchial aspiration biopsy identified a lung adenocarcinoma. Appropriate staging exams were compatible with T3N2M1 (stage IV).

The patient was submitted to the indicated chemotherapy, with partial response followed by disease relapse without response to second and third line chemotherapy. The patient died 3 years after the diagnosis. As for this dermatosis, the lesions followed the disease course, with slight initial improvement and relapse along with the oncologic disease.

\section{DISCUSSION}

Paraneoplastic syndromes are signs or symptoms that occur as a result of organ or tissue damage at locations remote from the site of the primary tumor or metastases. A wide variety of paraneoplastic syndromes are associated with lung adenocarcinoma. The symptoms often precede the diagnosis of the associated lung cancer, especially when the symptoms are neurologic or dermatologic, and the improvement generally occurs with targeted treatment of the underling tumor.?

Considering the rarity of the dermatosis in the general population, the concurrent onset and similar clinical course of both conditions, this case of NV is suggestive of a paraneoplastic manifestation of the lung adenocarcinoma.

Received 11 October 2017

Accepted 22 January 2018

* Work conducted at the Service of Dermatology and Venereology, Hospital Santo António dos Capuchos, Centro Hospitalar de Lisboa Central, Lisbon, Portugal. Financial support: None. Conflict of interest: None.

1 Department of Dermatology and Venereology, Hospital Santo António dos Capuchos, Centro Hospitalar de Lisboa Central, Lisbon, Portugal.

MAILING ADDRESS:

Ana Sofia Ribeiro Borges

E-mail: asofiarborges@gmail.com

(C2018 by Anais Brasileiros de Dermatologia 

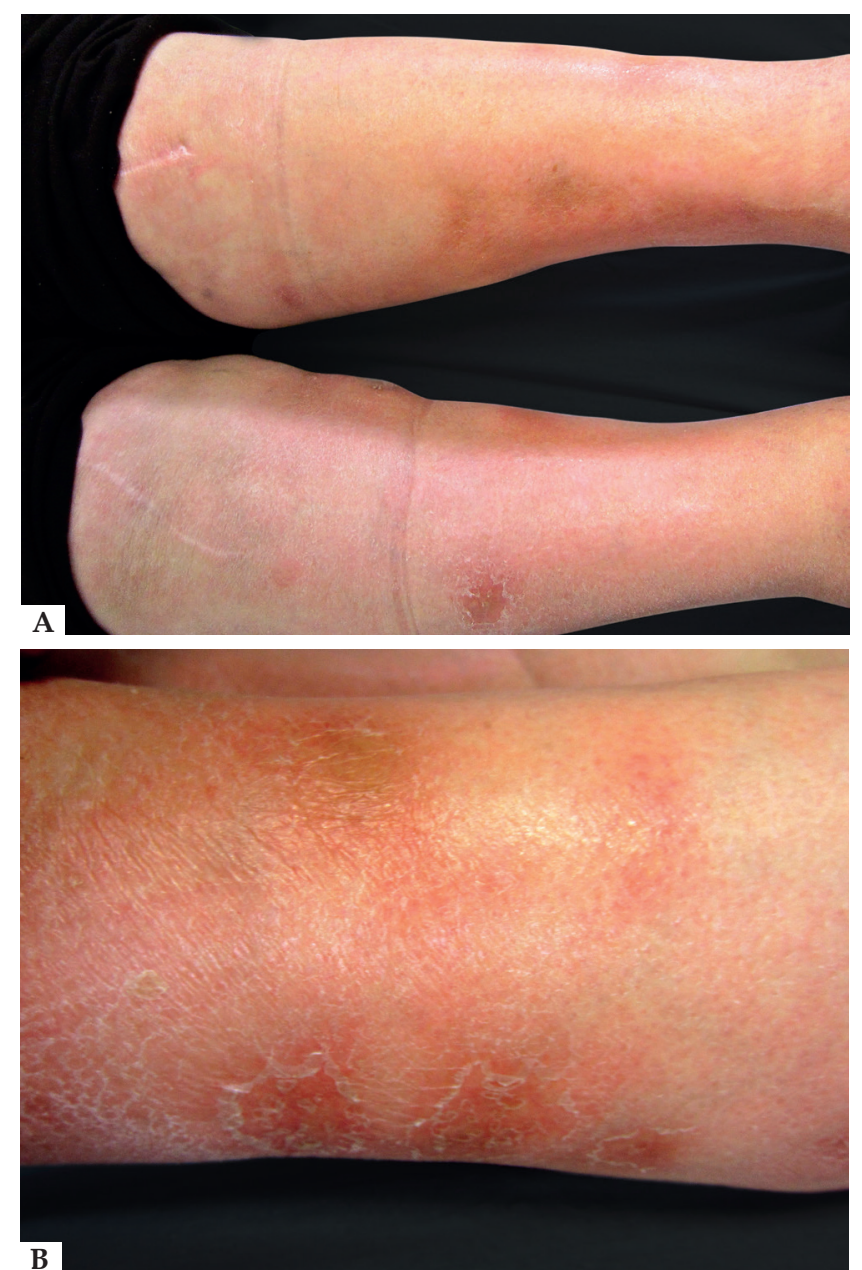

Figure 1: A AND B - Bilateral tender erythematous subcutaneous nodules on the lower legs

The physiopathology of paraneoplastic syndromes is not clear, however, some authors proposed an aberrant release of humoral mediators, hormones and hormone-like peptide by the tumor as the main pathogenic mechanism. ${ }^{7}$ Additionally, NV should be regarded as a delayed hypersensitivity response to a variety of agents, in which vasculitis, a histologic hallmark, could be the consequence of a cell-mediated cytotoxicity target to circulating immune complexes. Such findings support the hypothesis that NV could be a paraneoplastic skin manifestation due to circulating tumoral antigens.
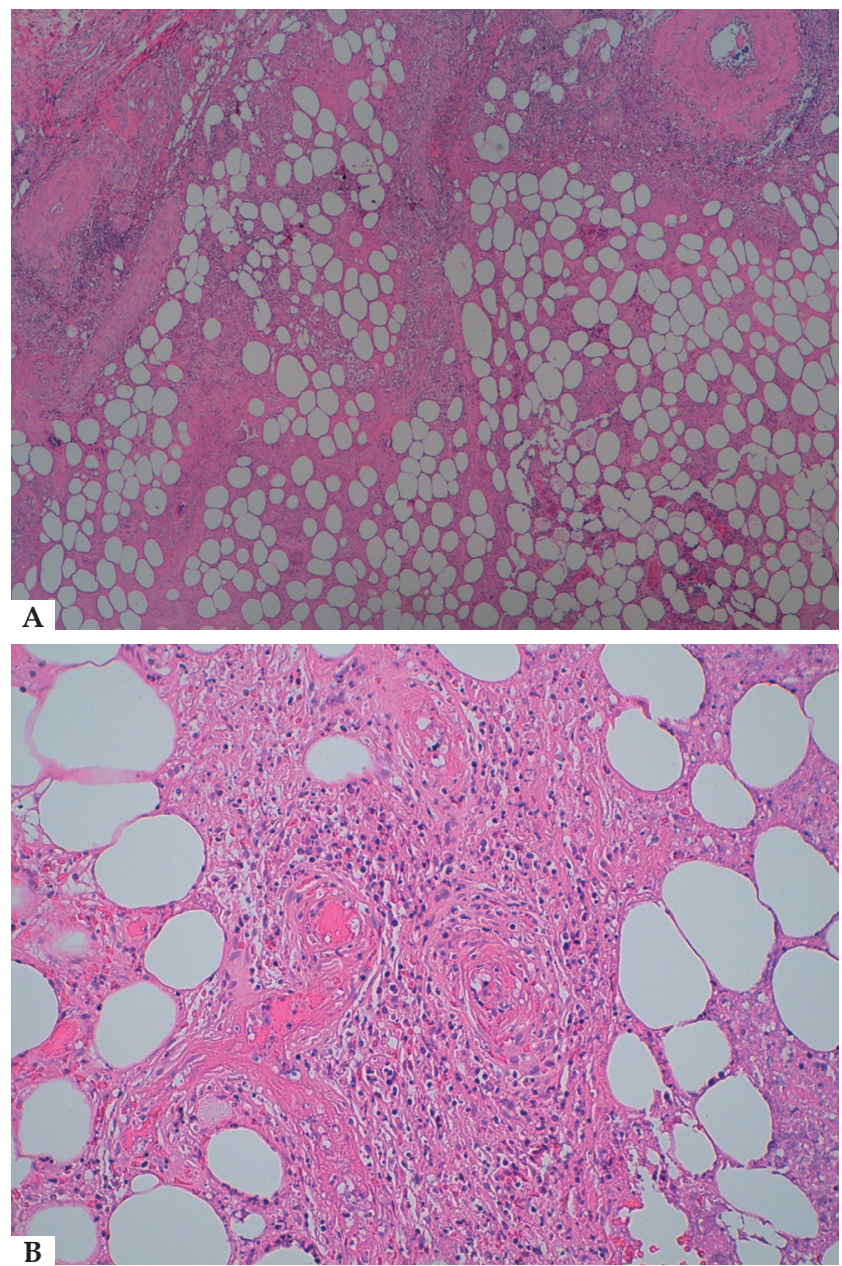

FIGURE 2: A - Histopathologic specimen showing predominantly lobular panniculitis with adipocyte necrosis (Hematoxylin \& eosin, x40); B- Vasculitis of hypodermal vessel with surrounding mixed infiltrate of neutrophils, lymphocytes, and histiocytes (Hematoxylin \& eosin, $\mathrm{x} 100)$

The early recognition of paraneoplastic syndromes may contribute to the detection of a highly treatable, early-stage tumor. Therefore, the identification of $\mathrm{NV}$ as the initial presentation of a tumor could be very important for the prognosis of the patient. On the other hand, this case reported emphasizes the need for more studies to understand the pathogenesis of NV. $\square$ 


\section{REFERENCES}

1. Segura S, Pujol RM, Trindade F, Requena L. Vasculitis in erythema induratum of Bazin: a histopathologic study of 101 biopsy specimens from 86 patients. J Am Acad Dermatol. 2008;59:839-51.

2. Gilchrist H, Patterson JW. Erythema nodosum and erythema induratum (nodular vasculitis): diagnosis and management. Dermatol Ther. 2010;23:320-7.

3. Misago N, Narisawa Y. Erythema induratum (nodular vasculitis) associated with Crohn's disease: a rare type of metastatic Crohn's disease. Am J Dermatopathol. 2012;34:325-9.
4.

Park SB, Chang IK, Im M, Lee Y, Kim CD, Seo YJ, et al. Nodular Vasculitis That Developed during Etanercept (Enbrel) Treatment in a Patient with Psoriasis. Ann Dermatol. 2015;27:605-7.

5. Khachemoune A, Longo MI, Phillipis TJ. Nodular vasculitis as a paraneoplasic presentation? Int J Dermat. 2003;42:639-42.

6. Requena L, Sánchez Yus E. Panniculitis. Part II. Mostly lobular panniculitis. J Am Acad Dermatol. 2001;45:325-61.

7. Kanaji N, Watanabe N, Kita N, Bandoh S, Tadokoro A, Ishii T, et al. Paraneoplastic syndromes associated with lung cancer. World J Clin Oncol. 2014;5:197-223.

\section{AUTHORS'CONTRIBUTIONS}

Ana Sofia Borge

Elaboration and writing of the manuscript

Ana Brasileiro

(iD) ORCID 0000-0002-2965-5962

Elaboration and

Margarida Apetato

Critical review of the manuscript
(iD) ORCID 0000-0002-8359-6561

review of the literature

(iD) ORCID 0000-0002-6151-6030

How to cite this article: Borges AS, Brasileiro A, Apetato M. Nodular vasculitis associated with lung adenocarcinoma. An Bras Dermatol. 2018;93(6):887-9. 